\title{
Obesity Enhances the Conversion of Adipose-Derived Stromal/Stem Cells into Carcinoma-Associated Fibroblast Leading to Cancer Cell Proliferation and Progression to an Invasive Phenotype
}

\author{
Amy L. Strong, ${ }^{1}$ Dorothy T. Pei, ${ }^{1}$ Christian G. Hurst, ${ }^{1}$ Jeffrey M. Gimble, ${ }^{2,3,4}$ \\ Matthew E. Burow, ${ }^{3}$ and Bruce A. Bunnell ${ }^{1}$ \\ ${ }^{1}$ Center for Stem Cell Research and Regenerative Medicine, Tulane University School of Medicine, New Orleans, LA, USA \\ ${ }^{2}$ LaCell LLC, New Orleans, LA 70112, USA \\ ${ }^{3}$ Departments of Medicine and Surgery, Tulane University School of Medicine, New Orleans, LA 70112, USA \\ ${ }^{4}$ Department of Medicine, Section of Hematology and Medical Oncology, Tulane Health Sciences Center, New Orleans, \\ LA 70112, USA
}

Correspondence should be addressed to Bruce A. Bunnell; bbunnell@tulane.edu

Received 25 February 2017; Revised 6 September 2017; Accepted 1 October 2017; Published 17 December 2017

Academic Editor: Heinrich Sauer

Copyright ( 2017 Amy L. Strong et al. This is an open access article distributed under the Creative Commons Attribution License, which permits unrestricted use, distribution, and reproduction in any medium, provided the original work is properly cited.

\begin{abstract}
Obesity is associated with enhanced tumor growth and progression. Within the adipose tissue are adipose-derived stromal/stem cells (ASCs) that have been shown to convert into carcinoma-associated fibroblast (CAFs) in the presence of tumor-derived factors. However, the impact of obesity on the ASCs and on the conversion of ASCs into CAFs has not been demonstrated. In the current study, ASCs isolated from lean donors (BMI $<25$; lnASCs) were compared with ASCs isolated from obese donors (BMI > 30, obASCs). The contribution of tumor-derived factors on the conversion of ASCs to CAFs was investigated. Following exposure to cancer cells, obASCs expressed higher levels of CAF markers, including NG2, alpha-SMA, VEGF, FAP, and FSP, compared to lnASCs. To investigate the crosstalk between ASCs and breast cancer cells, MCF7 cells were serially cocultured with lnASCs or obASCs. After coculture with lnASCs and obASCs, MCF7 cells demonstrated enhanced proliferation and expressed an invasive phenotype morphologically, with more pronounced effects following exposure to obASCs. Long-term exposure to obASCs also enhanced the expression of protumorgenic factors. Together, these results suggest that obesity alters ASCs to favor their rapid conversion into CAFs, which in turn enhances the proliferative rate, the phenotype, and gene expression profile of breast cancer cells.
\end{abstract}

\section{Introduction}

Adipose-derived stem/stromal cells (ASCs) are multipotent stromal cells isolated from adipose tissue and have been used for a wide variety of tissue engineering applications. Their multipotency, immunomodulatory properties, and regenerative potential have made ASCs an attractive candidate for clinical applications. However, studies have also shown the paradoxical effect of ASCs in promoting cancer $[1,2]$. Numerous studies have shown that soluble factors secreted by cancer cells reprogram ASCs to secrete growth factors, cytokines, and ECM-remodeling proteins, converting these cells into carcinoma-associated fibroblast- (CAF-) like cells [3-6]. CAFs display traits of myofibroblast and are abundant in the most invasive human breast cancers [7]. It has been shown that CAFs stimulate tumor growth and promote angiogenesis through the secretion of growth factors and proinflammatory cytokines, such as interleukins and interferons $[8,9]$. Moreover, CAFs alter the malignant potential of cancer cells by promoting the secretion of proinvasive factors, such as matrix metalloproteinases. Lastly, CAFs have been shown to alter the extracellular matrix of breast and 
adipose tissue. Differentiation of ASCs into CAFs results in the expression alpha-smooth muscle actin ( $\alpha$-SMA), fibroblast activation protein alpha (FAP1), and fibroblastspecific protein (FSP) in addition to the production of ECM proteins, such as collagens and fibronectin, which enhances the stiffness of cells [10]. Increased stiffness has been shown to promote the proliferation and metastasis of breast cancer cells $[11,12]$. Collectively, these studies have shown that ASCs may differentiate into CAFs and increase tumorigenesis, angiogenesis, cancer cell invasion and metastasis, resistance to chemotherapy, and cancer recurrence [13-19].

Obesity has reached epidemic levels in recent years and has become a major risk factor for breast cancer development $[20,21]$. In particular, studies have shown that a high body mass index (BMI) at disease onset has predictive value for poor prognosis in breast cancer patients [20, 21]. Excessive adipose tissue has been associated with an altered cytokine profile, characterized by a reduction in the release of antiinflammatory cytokines and an increased secretion of proinflammatory cytokines [22]. Consequently, obesity is considered to be an important source of low-grade chronic inflammation [23]. Prolonged exposure to high levels of proinflammatory cytokines secreted by adipose tissue before tumor formation may provide an environment conducive to the development and subsequent metastasis of breast cancer. Therefore, it is pertinent to determine the effects of obesity on ASC biology, as this may shed light on the precise mechanism(s) by which obesity may increase the morbidity and mortality of breast cancer.

Several studies investigating the effects of obesity on ASC biology have shown changes in the transcriptomic profile and immunomodulatory potential of ASCs isolated from obese subjects $[24,25]$. Additional studies have shown that coculture of these ASCs isolated from obese subjects (obASCs) results in increased tumor growth and metastasis of breast cancer cells $[26,27]$. While these previous studies have investigated the short-term effects of obASC exposure, few studies have investigated the effects of long-term exposure of obASCs on breast cancer cells and cancer cell proliferation. In the present study, we used serial coculture of obASCs with breast cancer cells to investigate the impacts on the ASCs and breast cancer cells. When compared to ASCs isolated from lean subjects (lnASCs), serial coculture with cancer cells resulted in the rapid and robust expression of CAF markers in obASCs. Analysis of cytokines secreted in the media during coculture revealed higher levels of cytokines secreted by obASCs. Further analysis of cancer cells revealed the rapid proliferation of cancer cells after long-term coculture with obASCs, whereas the effect was less pronounced following exposure to $\ln A S C s$. The results suggest that soluble factors produced by cancer cells induce the conversion of obASCs into CAFs more readily than lnASCs, leading to increased secretion of cytokines and chemokines from obASCs that reciprocally support the growth of cancer cells.

\section{Material and Methods}

2.1. Human Subjects. Human ASCs were obtained from 12 Caucasian females (2 groups, 6 donors per group) undergoing elective liposuction procedures, as previously described [28]. All protocols were reviewed and approved by the Pennington Biomedical Research Center Institutional Review Board, and all human participants provided written informed consent. Briefly, ASCs were isolated from processed lipoaspirates from the subcutaneous abdominal adipose tissue of lean or obese patients. Liposuction aspirates were incubated in $0.1 \%$ type I collagenase (Sigma) and $1 \%$ powdered bovine serum albumin (BSA, fraction V; Sigma) dissolved in $100 \mathrm{ml}$ of phosphate buffered saline (PBS) supplemented with $2 \mathrm{mM}$ calcium chloride. The mixture was placed in a $37^{\circ} \mathrm{C}$ shaking water bath or incubator at $75 \mathrm{rpm}$ for $60 \mathrm{~min}$ and then centrifuged to remove oil, fat, primary adipocytes, collagenase solution, and cellular debris. The resulting cell pellet was resuspended in stromal medium (SM), which consisted of Dulbecco's modified eagles medium (DMEM)/F12 (Hyclone, Logan UT), 10\% fetal bovine serum (Hyclone, Logan UT), and $1 \%$ antibiotic/antimycotic (Thermo Fisher Scientific, Waltham, MA), and plated in $175 \mathrm{~cm}^{2}$ flasks. Fresh SM was added every 2-3 days until cells achieved $80-90 \%$ confluence, at which time cells were harvested with $0.25 \%$ trypsin/1 mM EDTA (GIBCO, Thermo Fisher Scientific) and cryopreserved prior to experimental use. The mean BMI for the lnASC group was $22.7 \pm 1.9$, while the mean BMI for the obASCs was $32.7 \pm 3.7$. The mean age of the subjects for each group of donors was as follows: $38.8 \pm 7.0$ for $\ln$ ASCs and $42.5 \pm 8.9$ for obASCs. No statistical significance in age was observed between the donor groups.

\subsection{Cell Culture}

2.2.1. ASCs. Frozen vials of ASCs were thawed and cultured on $150 \mathrm{~cm}^{2}$ culture dishes (Nunc, Rochester, NY) in $25 \mathrm{ml}$ complete culture medium (CCM), which consisted of $\alpha$-minimal essential medium ( $\alpha$ MEM; GIBCO; Grand Island, NY), $20 \%$ fetal bovine serum (FBS; Atlanta Biologicals, Lawrenceville, GA), 100 units per $\mathrm{ml}$ penicillin $/ 100 \mu \mathrm{g} / \mathrm{ml}$ streptomycin (P/S; GIBCO), and $2 \mathrm{mM}$ L-glutamine (GIBCO). The cells were then incubated at $37^{\circ} \mathrm{C}$ with $5 \%$ humidified $\mathrm{CO}_{2}$. After 24 hours, viable cells were harvested with $0.25 \%$ trypsin/1 mM EDTA and replated at $100-200$ cells $/ \mathrm{cm}^{2}$ in CCM. The medium was changed every 2-3 days. For all experiments, subconfluent cells ( $\leq 70 \%$ confluent) between passages 2-6 were used. Characterization of stem cells was previously performed and published [26].

2.2.2. Breast Cancer Cell (BCC) Lines. MCF7 (HTB-22) cells were obtained directly from American Type Culture Collection (ATCC; Manassas, VA) and used for fewer than 6 months after resuscitation. MCF7 cells were chosen for the specific purpose that they represent a luminal-type breast cancer. Cell line authentication was conducted by ATCC via short tandem repeat profiling. Cells were cultured in Dulbecco's modified eagle's medium (DMEM; GIBCO), supplemented with $10 \% \mathrm{FBS}$ and $\mathrm{P} / \mathrm{S}$. Cells were grown at $37^{\circ} \mathrm{C}$ with $5 \%$ humidified $\mathrm{CO}_{2}$, fed every 2-3 days, and split $1: 4$ to $1: 6$ when the cells reached $90 \%$ confluence. We followed the methods of Strong et al. [27]. 
2.3. Generation of $G F P^{+} M C F 7$ Cells and $R F P^{+}$ASCs. To produce lentivirus, 293T cells were transfected through a modified calcium chloride transfection protocol when cells reached $70-75 \%$ confluence. For each transfection, $10 \mu \mathrm{g}$ of packaging plasmid, enveloping encoding plasmid, and transfer plasmid containing GFP and neomycin resistance or dsRed and neomycin resistance were used. After 48 hours, the medium was harvested and used to transduce cancer cells. To transduce MCF7 cells, conditioned medium containing virus with GFP and neomycin resistance was added to MCF7 cells at 70\% confluence. To transduce ASCs, conditioned medium containing virus with dsRed and neomycin resistance was added to ASCs at 70\% confluence. MCF7 cells and ASCs were selected with $500 \mu \mathrm{g} / \mathrm{ml}$ of Geneticin (Invitrogen; Carlsbad, CA) for 2 weeks and GFP expression or dsRed expression was verified with flow cytometry. All MCF7 cells and ASCs used for experimentation expressed GFP and dsRed, respectively. ASCs were characterized based on cell surface marker profile, colony-forming unit assay, and differentiation capacity as shown previously [26].

2.4. Proteome Profiler Cytokine Array. MCF7 cells were cultured alone or cocultured with InASCs or obASCs in DMEM medium containing 10\% FBS and P/S. After 7 days, the conditioned media was collected from the three conditions (MCF7, MCF7 with lnASCs, and MCF7 with obASCs) and stored in aliquots at $-80^{\circ} \mathrm{C}$ prior to experimentation. The Proteome Profiler Human XL Cytokine Array (R\&D Systems, Minneapolis, MN) was used according to manufacturer's instructions to detect 102 different cytokines directly in the biological replicates. Briefly, the conditioned media was added to cocktail of biotinylated antibodies and incubated at room temperature for 1 hour. The sample antibody mixture was subsequently incubated at $4^{\circ} \mathrm{C}$ for 19 hours with a membrane embedded with antibodies specific to the cytokines analyzed. Following a washing step, $3 \mathrm{ml}$ of a 1:1000 dilution of secondary antibody conjugated with streptavidin-HRP was added to each membrane and incubated at room temperature for 45 minutes. For detection, the membranes were visualized with chemiluminescence reagent provided with the kit on an ImageQuant LAS 4000 (GE Healthcare Life Science; Piscataway, NJ). Quantitative analysis of the protein array was conducted with densitometry.

2.5. MCF7 Cell and ASC Serial Coculture. MCF7 cells were cocultured with $\operatorname{lnASCs}(n=6$ donors) or obASCs ( $n=6$ donors) in a $1: 1$ ratio for a total of 100,000 cells in DMEM supplemented with $10 \%$ FBS and P/S. After 7 days, cocultured cells were harvested, washed, and FACS sorted with the Becton Dickinson FACSVantage SE Cell Sorter with DiVa option (BD, Franklin Lakes, NJ) based on dsRed expression (ASCs). After one coculture, cells were denoted with $\mathrm{cl}$, for example, cancer cells following the initial coculture would be denoted $\operatorname{lnMCF} 7(\mathrm{c} 1)$ or obMCF7(c1). Cells serially cocultured two times (c2) were generated from naïve MCF7 cells cocultured with $\operatorname{lnASC}(\mathrm{c} 1)$ or obASC(c1). After 7 days, these serially cocultured cells were FACS sorted, enriching for $\ln \mathrm{ASC}(\mathrm{c} 2)$ or $\mathrm{obASC}(\mathrm{c} 2)$. To generate serially cocultured MCF7 cells, naïve lnASCs were cocultured with $\operatorname{lnMCF} 7(\mathrm{c} 1)$ and naïve obASCs were cocultured with obMCF7(c1). After 7 days, these serially cocultured cells were sorted into lnMCF7(c2) and obMCF7(c2). Serial cocultures with the cancer cells were conducted until c4. Naïve MCF7 cells, naïve $\ln A S C s$, and naïve obASCs without previous coculture were collected and served as controls.

2.6. RNA Isolation Followed by Reverse Transcriptase Polymerase Chain Reaction ( $q R T-P C R$ ). Serially cocultured and FACS sorted MCF7 cells, InASCs, or obASCs were analyzed by qRT-PCR. RNA was extracted using TRIzol reagent (Invitrogen), purified with RNeasy columns (Qiagen), and digested with DNase I (Invitrogen). A total of $2 \mu \mathrm{g}$ of cellular RNA was used for cDNA synthesis with SuperScript VILO cDNA synthesis kit (Invitrogen). Quantitative real-time PCR was performed using the EXPRESS SYBR GreenER qPCR SuperMix Kit (Invitrogen) according to the manufacturer's instructions. Primer sequences used are located in Table 1 . At the completion of the reaction, $\Delta \Delta \mathrm{Ct}$ was calculated to quantify mRNA expression.

2.7. Alamar Blue Cell Proliferation Assay. Alamar blue cell proliferation assay was conducted according to the manufacturer's instructions. Briefly, 100 cells were sorted into a 96well plate in triplicates with FACS CloneCyte device and software (Becton Dickinson). After the cells adhered overnight, the medium was removed, the wells were washed with PBS, and the cells were incubated in 10\% Alamar blue reagent (Invitrogen). After overnight culture, the fluorescence intensity was measured at an excitation wavelength of $540 \mathrm{~nm}$ and an emission wavelength of $580 \mathrm{~nm}$ using a fluorescence plate reader (FLUOstar Optima; BMG Labtech Inc., Durham, NC). Cells were assessed on days 1, 2, 4, and 7.

2.8. Morphological Assessment. To assess the morphology of MCF7 cells after continuous exposure to $\ln$ ASCs or obASCs, FACS sorted MCF7(c1), $\operatorname{lnMCF7}(\mathrm{c} 1), \operatorname{obMCF7}(\mathrm{c} 1)$, MCF7(c4), lnMCF7(c4), and obMCF7(c4) were visualized with Nikon Eclipse TE200 (Melville, NY). Images were acquired on Nikon Digital Camera DXM1200F using the Nikon ACT-1 software version 2.7.

2.9. Statistical Analysis. All values are presented as mean \pm standard error of the mean (SEM). The statistical differences among three or more groups were determined by ANOVA, followed by post hoc Tukey multiple comparison tests versus the respective control group. Statistical significance was set at $P<0.05$. The analysis was performed using Prism (GraphPad Software, San Diego, CA).

\section{Results}

3.1. Obesity Alters the Secretome Profile of Cocultured Cells. The secretome profiles of MCF7 cells cultured alone and cocultured with $\operatorname{lnASC}$ or obASCs were assessed with the proteome profiler array. Of the 102 cytokines assessed, the array showed increased expression of 21 proteins in the cocultured samples: adiponectin, chitinase 3-like 1, complement factor D, CXCL5, endoglin/CD105, IGFBP-3, 
TABle 1: Primers for qRT-PCR.

\begin{tabular}{lcc}
\hline Gene & Forward $\left(5^{\prime} \rightarrow 3^{\prime}\right)$ & Reverse $\left(5^{\prime} \rightarrow 3^{\prime}\right)$ \\
\hline Cytokines & Adiponectin & \\
Chitinase 3-like 1 & TGTGAACCCATTCGCTTTAC & AGAGGCTGACCTTCACATCC \\
Complement factor D & GGTCACCCAAGCAACAAAGT & TACAGAGGAAGCGGTCAAGG \\
IGFBP-3 & TAAGTATGGGCAGCCTCTCC & CCATGCTGATCTCGAACTCC \\
IL-6 & GTAGCCGCCCCACACAGACAGCC & GGTCATGTCCTTGGCAGTCT \\
Leptin & GAAGACCACATCCACACACG & GCCATCTTTGGAAGGTTC \\
IL-24 & GCCTCTGATTGGTGAATGGT & AGCTCAGCCAGACCCATCTA \\
Pentraxin-3 & ATTCAGAGGAAGGGCTCACA & GGTGTTAAATTGGCGAAAGC \\
CCL5 & CGCTGTCATCCTCATTGCTA & TGTTTCATCAAAGCCACCAC \\
SerpinE1 & CAGACCAAGAGCCTCTCCAC & GCACTTGCCACTGGTGTAGA \\
CCL17 & CACCCCAGACTCCTGACTGT & GACTGTTCCTGTGGGGTTGT \\
CAF markers & CATGGCTCCAGTTCAGACAA \\
PDGFR $\beta$ & GCACTTTTATCCACCCAGGA & \\
NG2 & AGTATGGGCATCTCCTGGTG & \\
-SMA & AATGGCTCTGGGCTCTGTAA & GTACTTGGCTCAGCCTCCAG \\
FAP & CGAGGGCCTGGAGTGTGT & CATTGACACCCCTAGCCAGT \\
FSP & TACCCAAAGGCTGGAGCTAA & TTTGCTCTGTGCTTCGTCAC \\
\hline Housekeeping gene & CAAGTACTCGGGCAAAGAGG & CCGCATAATCTGCATGGTGAT \\
$\beta$-actin & ACAGGACCGAAACATTCTGG \\
\hline CA $=$ CACCTTCTACAATGAGCTGC & TGCAGGACAGGAAGACACAG \\
\hline
\end{tabular}

$\mathrm{CAF}=$ carcinoma-associated fibroblast.

IL-4, IL-6, IL-16, IL-23, IL-24, IL-33, leptin, LIF, myeloperoxidase, osteopontin, pentraxin-3, CCL5/RANTES, serpinE1, CCL17/TARC, and UPAR. Of these 21 proteins, 11 factors were overexpressed in the MCF7/obASCs compared to the MCF7/lnASCs group: adiponectin (61.5-fold versus 8.0 -fold, $P<0.001)$, chitinase 3 -like 1 (117.8-fold versus 60.1-fold, $P<0.01)$, complement factor D $(3.3$-fold versus 1.2-fold, $P<0.01$ ), IGFBP-3 (7.3-fold versus 5.6-fold, $P<0.01$ ), IL-6 (8.1-fold versus 6.4-fold, $P<0.05$ ), IL-24 (18.4-fold versus 10.0 -fold, $P<0.05)$, leptin (27.5-fold versus 0.9 -fold, $P<0.001$ ), pentraxin-3 (4.1-fold versus 2.9-fold, $P<0.05)$, CCL5/RANTES (4.2-fold versus 1.7 -fold, $P<0.01$ ), serpinE1 (23.8-fold versus 18.1 -fold, $P<0.05)$, and CCL17/ TARC (3.0-fold versus 1.3-fold, $P<0.001$ ) (Figure 1).

3.2. Serial MCF7 Coculture Leads to More Robust Expression of CAF Markers in obASCs Compared to InASCs. To determine whether the rate of conversion of ASCs to CAFs varies between $\ln$ ASCs and obASCs following exposure to cancer cells, we investigated the expression of CAF markers in ASCs temporally. Serial coculture with MCF7 cells enhanced the gene expression of PDGFR-beta in lnASCs and obASCs; however, no statistically significant difference was observed between InASCs and obASCs. In contrast, following coculture with MCF7 cells, the expression of several genes in obASCs was enhanced compared to lnASCs: NG2 (2.3-fold in obASCs and 1.2-fold in lnASCs, $P<0.001), \alpha$-SMA (2.1-fold in obASCs and 1.0-fold in lnASCs, $P<0.01)$, VEGF $(3.2$-fold in obASCs and 1.3 -fold in lnASCs, $P<0.01)$, FAP1 $(3.2$-fold in obASCs and 1.3 - fold in $\ln$ ASCs, $P<0.001$ ), and FSP (3.1-fold in obASCs and 0.9 -fold in $\ln$ ASCs, $P<0.001$; Figure 2). Following two serial cocultures, obASCs (2.2-fold) continued to demonstrate expression of $\alpha$-SMA compared to lnASCs $(0.8$ fold, $P<0.001$; Figure 2). Interestingly, the expressions of FAP1 and FSP in InASCs were higher than those in obASCs after exposure to MCF7 for two serial cocultures: FAP1 (4.4-fold in lnASCs versus 2.5 -fold in obASCs, $P<0.001$ ) and FSP (2.8-fold in lnASCs versus 1.6-fold in obASCs, $P<0.05$; Figure 2).

3.3. Serial Coculture with MCF7 Cells Leads to Enhanced Expression of Cytokines in obASCs Compared to InASCs. Further analysis was conducted in lnASCs and obASCs before coculture to determine if these cells express different levels of cytokines at baseline and to determine whether the stem cells or the cancer cells were secreting the cytokines. The expressions of the following cytokines were differentially expressed between lnASCs and obASCs at baseline: complement factor D (1.0-fold in lnASCs; 0.4 -fold in obASCs, $P<0.001)$, IL-6 (1.0-fold in $\ln$ ASCs and 2.1-fold in obASCs, $P<0.001)$, leptin $(1.0$-fold in lnASCs and 134.9-fold in obASCs, $P<0.001$ ), pentraxin3 (1.0-fold in lnASCs and 0.5 -fold in obASCs, $P<0.001$ ), CCL5/RANTES (1.0-fold in lnASCs and 1.7-fold in obASCs, $P<0.001)$, and serpineE1 $(1.0$-fold in $\ln$ ASCs and 0.6 -fold in obASCs, $P<0.001)$. No statistically significant difference was observed in adiponectin, chitinase 3-like 1, IGFBP-3, IL-24, and CCL17/TARC. Following the initial coculture with MCF7 cells, expression of these 


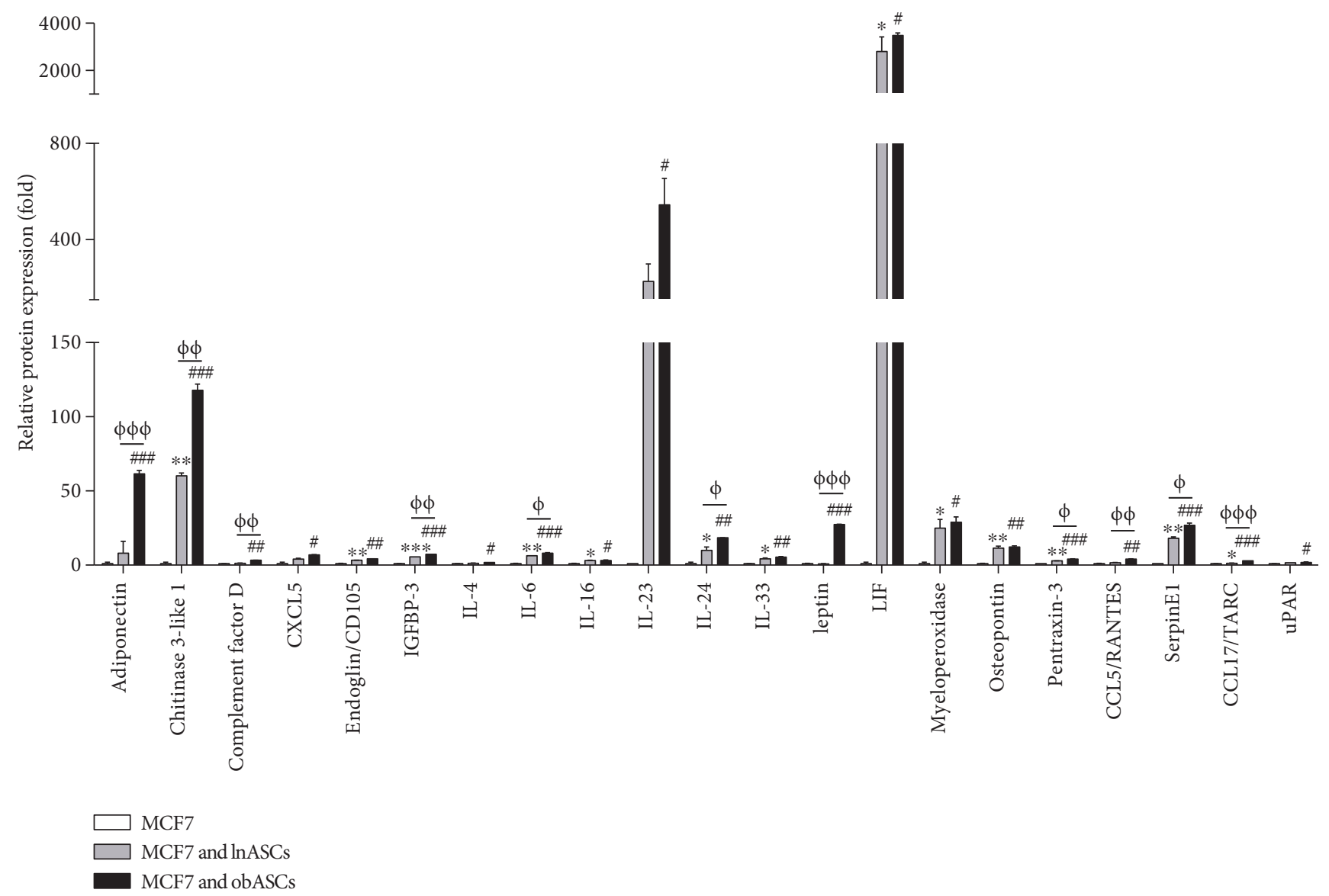

FIgURE 1: Secretome of MCF7 cells differs from secretome of MCF7 cells cocultured with lnASCs and obASCs. MCF7 cells were cultured alone or cocultured with lnASCs or obASCs for 7 days. The levels of various factors in the supernatants were measured by Proteome Profiler Cytokine Array at day 7 and were normalized to the levels observed in the media of MCF7 cells cultured alone. Bar: \pm SEM. ${ }^{*} P<0.05$; ${ }^{* *} P<0.01$; ${ }^{* * *} P<0.001$ between MCF7 and MCF7/lnASCs. ${ }^{\#} P<0.05$; \#\# $P<0.01$; \#\#\# $P<0.001$ between MCF7 and MCF7/obASCs. ${ }^{\Phi} P<0.05 ;{ }^{\Phi \Phi} P<0.01$; ${ }^{\Phi \Phi \Phi} P<0.001$ between MCF7/lnASCs and MCF7/obASCs.

cytokines and additional cytokines was reduced in $\ln \mathrm{ASCs}(\mathrm{c} 1)$ compared to obASCs$(\mathrm{c} 1)$ : 0.2 -fold and 0.7 fold for chitinase 3-like $1 \quad(P<0.001), 0.3$-fold and 1.0-fold for IL-6 $(P<0.001), 3.5$-fold and 26.2-fold for leptin $(P<0.001), 0.4$-fold and 0.8 -fold for pentraxin-3 $(P<0.001), 0.2$-fold and 0.6 -fold for CCL5 $(P<0.001)$, and 0.6 -fold and 1.2 -fold for serpinE1 $(P<0.001)$ in lnASCs and obASCs, respectively (Figure 3). Following a second round of coculture, the expression of IL-24 was significantly upregulated in obASCs(c2) to 10.1-fold, whereas $\ln$ ASCs(c2) expressed 0.8-fold of IL-24 $(P<0.001$; Figure 3$)$. Levels of chitinase 3 -like 1 (2.6-fold in $\operatorname{lnASCs}(\mathrm{c} 2)$ versus 1.4 -fold in obASCs(c2), $P<0.001$ ), complement factor $\mathrm{D}$ (1.8-fold in $\ln \mathrm{ASCs}(\mathrm{c} 2)$ versus 0.7 -fold in obASCs(c2), $P<0.001)$, and pentraxin-3 (0.9-fold in $\ln A S C s(c 2)$ versus 0.6 -fold in obASCs(c2), $P<0.001)$ were upregulated in $\ln$ ASCs(c2) compared to obASCs(c2) (Figure 3). These findings suggest that while levels of induction are important, the temporal expression is equally important. obASCs demonstrated higher levels of these factors following the initial coculture, while lnASCs required two serial cocultures before the expression was increased. Correlating the findings between the secretome and the gene expression profiles, the results indicate that while several key factors are secreted by ASCs, other cytokines are secreted by cancer cells following coculture.

3.4. Serial Coculture with obASCs Leads to More Robust Expression of Cytokines in MCF7 Cells, Compared to InASCs. To monitor changes associated with long-term exposure of the cancer cells to InASC or obASCs, MCF7 cells were serially cocultured with lnASCs or obASCs and sorted. The 11 differentially expressed cytokines identified by the proteome profiler were analyzed further by qRT-PCR to determine whether the MCF7 cells, following serial coculture, were responsible for the enhanced secretion of several cytokines. After the initial coculture with lnASCs or obASCs, MCF7 cells demonstrated enhanced expression of several cytokines, with a more robust response following exposure to obASCs: IGFBP-3 (10.9-fold in $\operatorname{lnMCF} 7(\mathrm{c} 1)$ and 158.8fold in obMCF7(c1), $P<0.001)$; IL-6 $(18.2$-fold in $\operatorname{lnMCF} 7(\mathrm{c} 1)$ and 84.7 -fold in obMCF7(c1), $P<0.001)$; serpinE1 (11.2-fold in $\operatorname{lnMCF} 7(\mathrm{cl})$ and 94.8 -fold in obMCF7(c1), $P<0.001$; Figure 4$)$. Levels of IGFBP-3 and IL-6 reduced to baseline after MCF7 cells were serially cocultured twice (Figure 4). IL-24 expression was higher in 

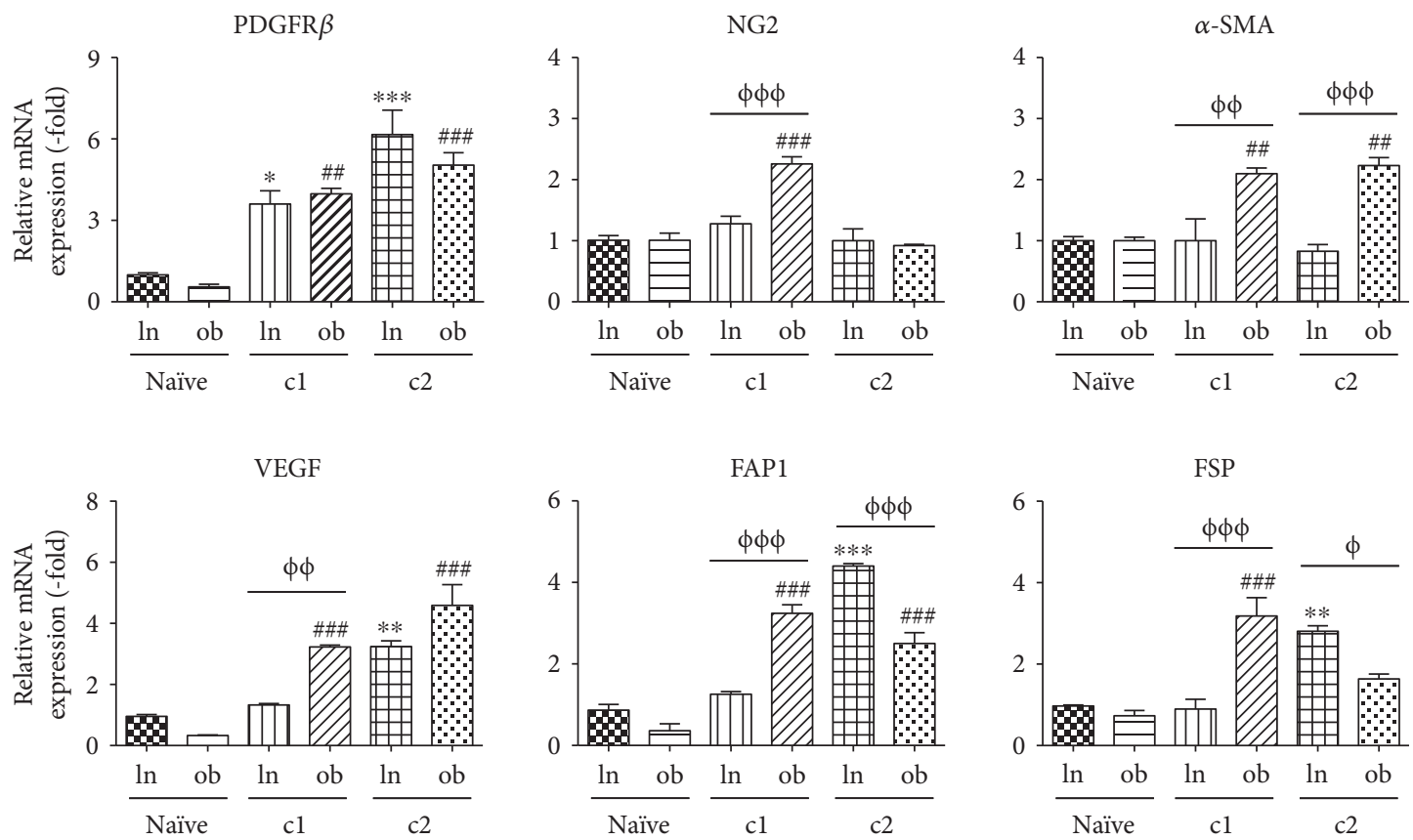

FIGURE 2: lnASCs and obASCs demonstrated enhanced expression of CAF markers following coculture. lnASCs and obASCs were serially cocultured with MCF7 cells. After 7 days, the cocultured cells (c1) were sorted, and the gene expression of various CAF markers in lnASCs and obASCs was assessed by qRT-PCR. The sorted $\operatorname{lnASC}(\mathrm{c} 1)$ or obASC(c1) cells were cocultured with MCF7 cells for another 7 days and sorted. Expression of CAF markers in lnASCs was assessed by qRT-PCR and normalized to the levels observed in lnASCs without exposure to MCF7 cells (naïve). Bar: \pm SEM. ${ }^{*} P<0.05 ;{ }^{* *} P<0.01 ;{ }^{* * *} P<0.001$ compared to naïve $\ln A S C s .{ }^{\#} P<0.01 ;{ }^{\# \# \#} P<$ 0.001 compared to naïve obASCs. ${ }^{\Phi} P<0.05 ;{ }^{\Phi \Phi} P<0.01 ;{ }^{\Phi \Phi \Phi} P<0.001$ between $\ln$ ASCs and obASCs.

lnMCF7(c2) cells $(16.0$-fold, $P<0.001)$ compared to obMCF7(c2) cells (12.3-fold, $P<0.001$; Figure 4$)$. Interestingly, long-term serial coculture with obASCs for four cocultures enhanced the expression of the following cytokines, compared to baseline and to MCF7 cells exposed to lnASCs: adiponectin (0.6-fold in $\operatorname{lnMCF} 7(\mathrm{c} 4)$ and 6.2 -fold in obMCF7 (c4), $P<0.001$ ), leptin (316.0-fold in lnMCF7(c4) and 1208.7-fold in obMCF7(c4), $P<0.001$ ), IL-24 (2.8-fold in $\operatorname{lnMCF} 7(\mathrm{c} 4)$ and 41.1-fold in obMCF7(c4), $P<0.001$ ), pentraxin-3 (0.9-fold in $\operatorname{lnMCF} 7(\mathrm{c} 4)$; 2.4 -fold in obMCF7(c4), $P<0.001$ ), and CCL5 (0.3-fold lnMCF7(c4) and 2.6-fold obMCF7 (c4), $P<0.001$; Figure 4). Other patterns of expression included upregulation of CCL17/TARC in MCF7 cells following one, two, and four serial cocultures with obASCs (11.8-fold in obMCF7(c1) and 11.8-fold in obMCF7(c2) and 10.5-fold in obMCF7(c4); $P<0.001)$. Several genes also demonstrated diminished gene expression levels following one or two serial cocultures, such as complement factor D and CCL5 (Figure 4). These results indicate the temporal effects of serial coculture on gene expression of cytokines. Following exposure to lnASCs, an absent or blunted effect in the gene expression was observed in MCF7s as compared to obASCs.

3.5. Serial Coculture with ASCs Alters Cancer Cell Gene Expression, Cell Proliferation, and Invasive Phenotype. To assess the proliferative effects of coculture on MCF7 cells, FACS sorted MCF7 cells that were serially cocultured alone, with $\operatorname{lnASCs}$, or with obASCs were evaluated with Alamar
Blue. Exposure to $\ln$ ASCs and obASCs after one coculture enhanced the proliferation of MCF7 cells. $\operatorname{lnMCF} 7(\mathrm{c} 1)$ cells (1090 RDU; $P<0.001)$ and obMCF7(c1) cells (1797 RDU, $P<0.001)$ demonstrated enhanced proliferation compared to the MCF7 cells cultured alone (736 RDU), when assessed on day 7 (Figure 5). After four serial cocultures with lnASCs and obASCs, MCF7 cells continued to demonstrate enhanced proliferation (702 RDU in MCF7 and 2085 RDU in lnMCF7(c4) and 2759 RDU in obMCF7(c4), $P<0.001)$. Furthermore, the proliferative effects of longterm coculture of obASCs on MCF7 cells were more robust than the long-term effects of serial coculture with $\ln$ ASCs over four cocultures $(P<0.001$; Figure 5$)$.

To determine whether serial coculture with $\ln$ ASCs and obASCs altered the cancer cell morphology, cocultured MCF7 cells were sorted and visualized under brightfield microscopy. No significant visible difference was observed in MCF7 cells after the first coculture with lnASCs. The majority of the MCF7 cells cocultured with obASCs maintained the epithelial morphology, while a few sporadic cells displayed more mesenchymal-like phenotype after the initial coculture. After four serial cocultures, MCF7 cells cultured with obASCs displayed even more mesenchymal-like phenotype, with increased invadopodia and increased phenotypical heterogeneity (Figure 6). MCF7 cells cultured alone or serially cocultured with $\ln$ ASCs maintained a less invasive phenotype (Figure 6). These results suggest that continuous exposure to obASCs enhances cancer cell proliferation and phenotypical heterogeneity, as compared to exposure to 

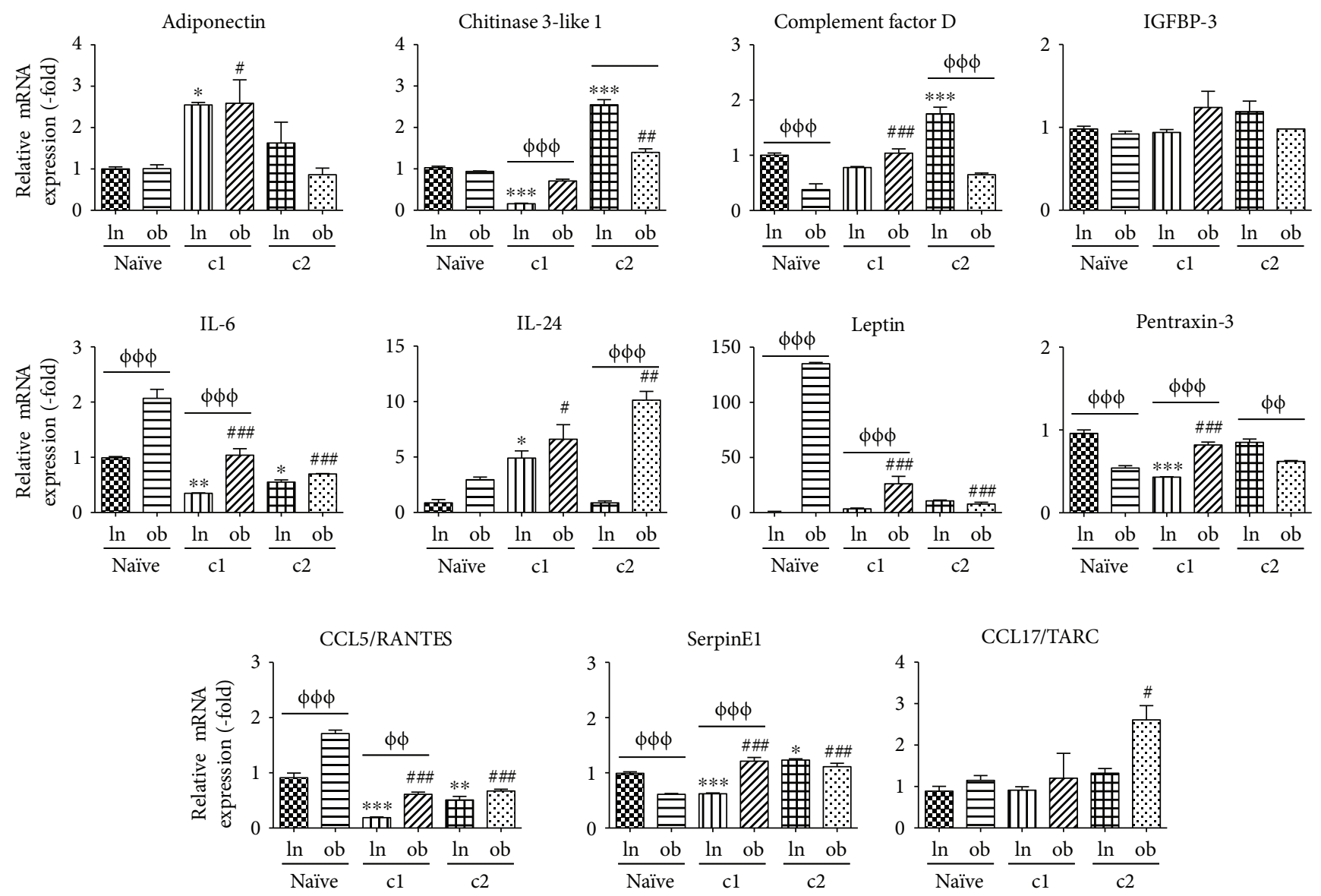

Figure 3: $\ln$ ASCs and obASCs demonstrated enhanced expression of cytokines following serial coculture. lnASCs and obASCs were serially cocultured with MCF7 cells. After 7 days, the cocultured cells (c1) were sorted, and the gene expression of various factors in lnASCs and obASCs was assessed by qRT-PCR. The sorted $\operatorname{lnASC}(\mathrm{c} 1)$ or obASC(c1) cells were cocultured with MCF7 cells for another 7 days and sorted. The gene expression of various factors in lnASCs was assessed by qRT-PCR and normalized to the levels observed in lnASCs without exposure to MCF7 cells (naïve). Bar: \pm SEM. ${ }^{*} P<0.05 ;{ }^{* *} P<0.01 ;{ }^{* * *} P<0.001$ compared to naïve $\ln A S C s$. ${ }^{\#} P<0.05$; ${ }^{\# \#} P<0.01$; ${ }^{\# \# \#} P<0.001$ compared to naïve obASCs. ${ }^{\Phi \Phi} P<0.01 ;{ }^{\Phi \Phi} P<0.001$ between $\ln$ ASCs and obASCs.

lnASCs or cultured alone, indicating that obASCs may play a role in enhancing tumor progression.

\section{Discussion}

Obesity is a significant health issue worldwide, particularly in light of its association with increased risk for a myriad of diseases, including breast cancer. The rapid expansion of fat tissue results in the release of several key cytokines and adipokines involved in chronic inflammation [29]. This local chronic inflammation has the potential to alter cells within the microenvironment of the adipose tissue, including the ASCs. In the current study, lnASCs and obASCs were cocultured with cancer cells to assess their respective effects on the secretome profile of breast cancer cells. Furthermore, the plasticity of ASCs to convert into CAFs and their effects on cancer cells were assessed after long-term serial coculture. Compared to lnASCs cocultured with cancer cells, obASCs cocultured with cancer cells demonstrated a more robust increase in the expression of several key cytokines that have been linked to aggressive breast cancers. Long-term coculture with obASCs resulted in increased proliferation of breast cancer cells. These findings suggest that changes in the local adipose stromal microenvironment in obese subjects impact the biology of ASCs and that these obASCs have an increased capacity to alter breast cancer proliferation and invasion.

To determine whether the increased expression of these key cytokines was associated with an increased rate of conversion into CAFs, lnASCs and obASCs were serially cocultured with MCF7 cells and assessed for the expression of CAF markers. obASCs expressed higher levels of CAF markers after the initial coculture, whereas InASCs expressed some of the CAF markers only after the second coculture. Several studies have reported that ASCs are a significant source of CAFs and their differentiation into CAFs is driven under the influence of tumor-derived factors [30]. Once converted into CAFs, these cells have been shown to play a central role in regulating inflammation and in promoting proliferation, migration, and angiogenesis of several cancers [31]. The results from our study suggest that obASCs have a higher propensity to convert into CAFs. These are consistent with previous findings that suggest that obASCs, 

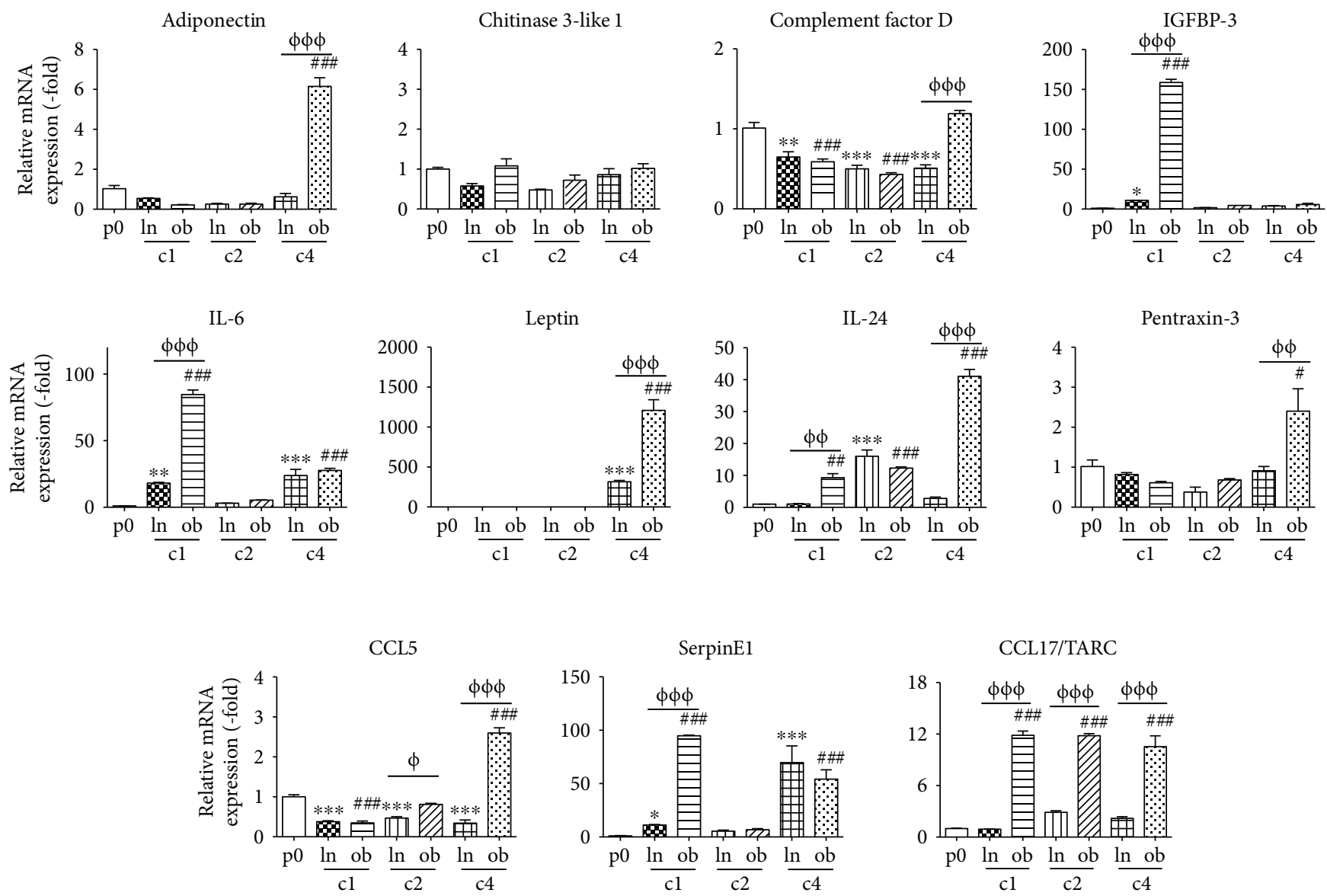

FIGURE 4: MCF7 cells demonstrated enhanced expression of cytokines following serial coculture. MCF7 cells were passaged or sorted after cocultured with lnASCs or obASCs for 7 days. The gene expression of various cytokines and chemokines in MCF7 cells was assessed by qRT-PCR prior to coculture (naïve) and after one coculture (c1). These lnMCF7(c1) and obMCF7(c1) cells were serially cocultured for another 7, 14, and 21 days to generate lnMCF7(c4) and obMCF7(c4) after four serial cocultures. This lnMCF7(c4) and obMCF7(c4) cells were sorted, and the expression of various factors was assessed by qRT-PCR and normalized to the levels observed in naïve MCF7 cells. Bar: \pm SEM. CC: coculture. ${ }^{\#} P<0.05$; ${ }^{\# \#} P<0.01$; ${ }^{\# \#} P<0.001$ between naive MCF7 and MCF7s exposed to obASCs. ${ }^{\Phi} P<0.05 ;{ }^{\Phi \Phi} P<0.01 ;{ }^{\Phi \Phi \Phi} P<0.001$ between MCF7 cells exposed to $\ln$ ASCs and MCF7 cells exposed to obASCs. ${ }^{*} P<0.05{ }^{* *} P<0.01$ and ${ }^{* * *} P<0.001$ between naive MCF7 and MCF7s exposed to lnASCs.
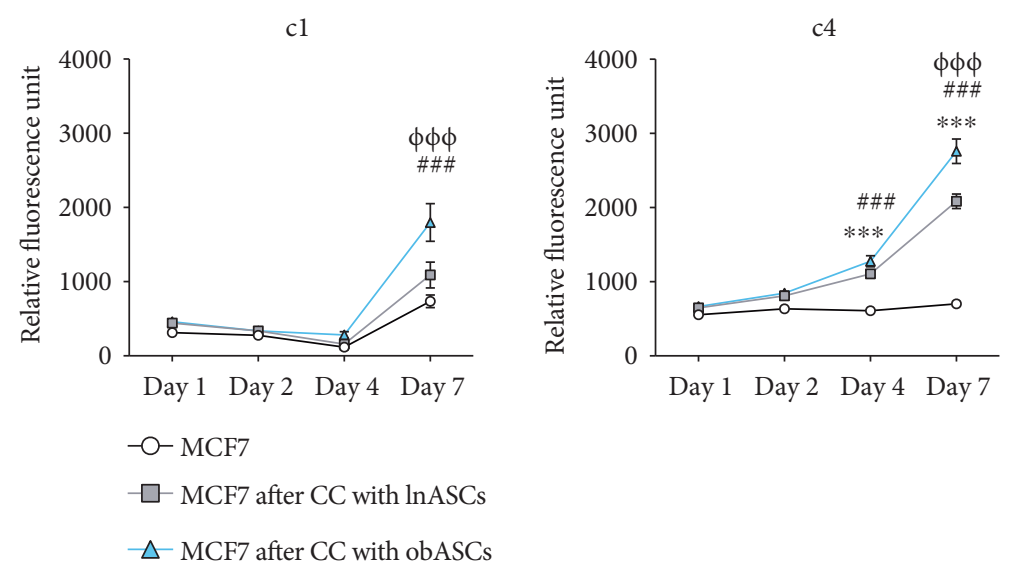

FIGURE 5: Serial coculture with obASCs enhances proliferation of breast cancer cells. MCF7 cells were serially cocultured with MCF7 cells. Sorted MCF7 cells were assessed by Alamar blue proliferation assay after one coculture ( 1$)$ and four cocultures (c4). Bar: \pm SEM. CC: coculture. ${ }^{* * *} P<0.001$ between MCF7 cells and MCF7 exposed to lnASCs. ${ }^{\# \#} P<0.001$ between MCF7 cells and MCF7 exposed to obASCs. ${ }^{\Phi \Phi} P<0.001$ between MCF7 cells exposed to lnASCs and MCF7 cells exposed to obASCs. 


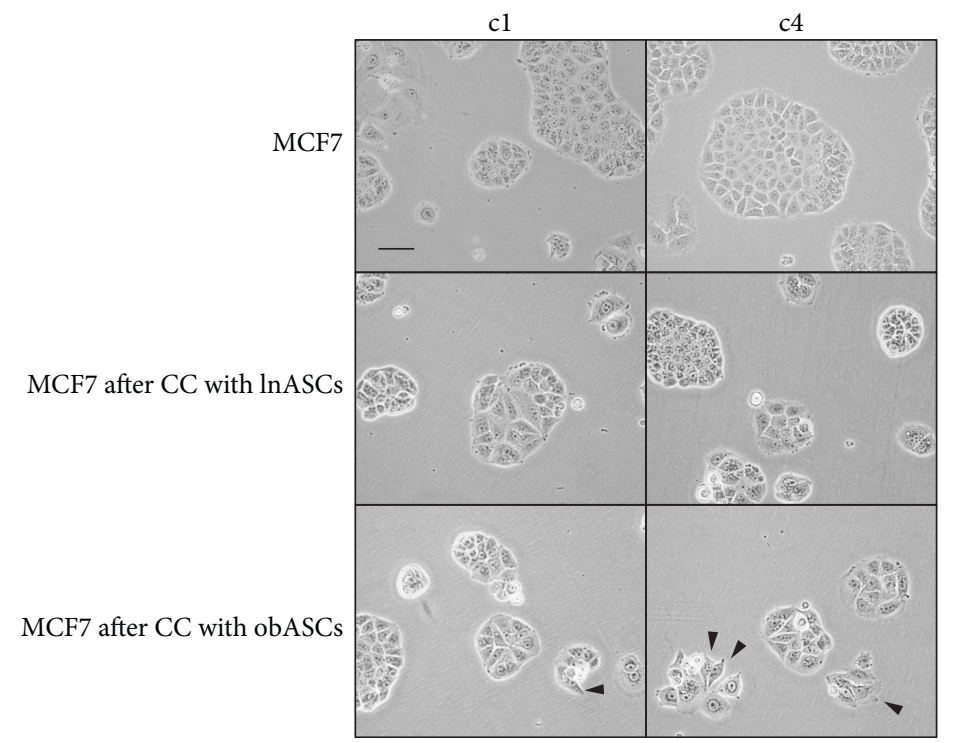

FIGURE 6: Serial coculture with obASCs enhances invasive phenotype of breast cancer cells. MCF7 cells were serially passaged or serially cocultured with lnASCs or obASCs and assessed morphologically. Arrowheads indicate more mesenchymal-like phenotype. Scale bar represents $200 \mu \mathrm{m}$.

conditioned by their proinflammatory environment, may acquire additional phenotypes that allow for increased migration and invasion [32].

Coculture of $\ln$ ASCs and obASCs with breast cancer cells resulted in the alteration in the secretome profile. However, to investigate which cell type was responsible for the altered expression levels of cytokines and to assess the effects of long-term coculture, InASCs and obASCs were assessed at baseline and following serially coculture with breast cancer cells for the cytokines identified by the secretome analysis. Baseline comparisons between InASCs and obASCs identified higher levels of IL-6, leptin, and CCL5 expression in obASCs compared to lnASCs. These factors all contribute to inflammation and have been shown to enhance tumor growth and metastasis [33-36]. Following continuous exposure to breast cancer cells, the expression of IL-24 in obASCs also continued to increase with each serial coculture, whereas the levels of IL-24 were significantly reduced in $\ln$ ASCs after the initial coculture. IL-24 has been shown to inhibit the growth of breast cancer cells through the activation of apoptotic pathways and the inhibition of angiogenesis [37, 38]. These results are inconsistent with our current findings and could be associated with differences in human and murine cells. The previously published studies generated breast cancer with the mouse mammary tumor virus and this specie difference could account for the observed discrepancy $[37,38]$. Furthermore, these studies validate the effects of IL24 receptor in triple-negative cell lines [37, 38], rather than the estrogen receptor-positive and progesterone receptorpositive cell line used in the current study, which partially explains the differences observed. Nevertheless, the contrast between our findings and those in previous reports indicates that additional analysis into the role of IL-24 in obASCs through inhibition or knockdown studies would shed light on the effects of IL-24 on breast cancer cells.
Interestingly, breast cancer cells also expressed high levels of cytokines following coculture with lnASCs or obASCs. The gene induction following the initial coculture with obASCs was significantly more robust, particularly in the expression of IL-6, IGFBP-3, and serpinE1. IL-6 has been shown to promote tumor survival, metastasis, and angiogenesis. Human primary mammospheres from node invasive breast cancers express higher levels of IL-6 than from mammospheres from matched nonneoplastic mammary glands [39]. Furthermore, breast cancer cells that were sensitive to drug treatment did not express IL-6, whereas multidrugresistant breast cancer cells produced high levels of IL-6 [40]. These findings support the data generated from a meta-analysis involving 3224 identified breast cancer patients where IL-6 expression was associated with poor prognosis [41]. Similarly, higher levels of IGFBP-3 have been associated with tumor progression and resistance to treatment due to the intranuclear roles of IGFBPs in transcriptional regulation, induction of apoptosis, and DNA damage repair [42]. Likewise, increased expression of serpinE1 has been correlated with tumor aggressiveness and poor clinical outcomes. The robust expression of IL-6, IGFBP-3, and serpinE1 following coculture with obASCs would suggest that these cancer cells are more aggressive. Furthermore, the enhanced expression of these factors correlated with a more invasive morphology and increased proliferation rate in breast cancer cells following coculture with obASCs compared to lnASCs. Interestingly, the expression of IGFBP-3 and serpinE1 was significantly higher in lnASCs after five serial cocultures, compared to obASCs after five serial cocultures. These findings suggest that lnASCs require significantly more conditioning with breast cancer cells to increase the expression of these factors, whereas obASCs only require the initial exposure to induce similar robust levels of expression. Collectively, these studies suggest that exposure 
to obASCs results in a more robust induction of cytokines in breast cancer cells and that induction is time dependent, whereby obASCs have the potential to increase the expression of these cytokines following fewer cocultures.

\section{Conclusion}

obASCs increase the proliferation and induce an invasive phenotype in breast cancer cells following long-term serial coculture. Following exposure to breast cancer cells, obASCs express higher levels of CAF markers at earlier time points, indicating that obASCs have a higher propensity to convert into CAFs. Furthermore, analysis of the cytokines and chemokines secreted during coculture demonstrate that breast cancer cells induce higher levels of cytokine expression in obASCs, compared to lnASCs. Moreover, exposure to obASCs results in a more robust level of induction in breast cancer cells. Future studies to inhibit the expression of these factors will shed light on the importance of these factors during breast cancer progression. Identifying the factors relevant to the disease progression will assist in developing targets to reduce the morbidity and mortality associated with breast cancer in obese women.

\section{Conflicts of Interest}

The first author and the corresponding author declare that there is no conflict of interest regarding the publication of this paper. Dr. Jeffrey M. Gimble is the coowner and CSO of LaCell LLC.

\section{Authors' Contributions}

Dorothy T. Pei and Christian G. Hurst contributed equally to this work.

\section{References}

[1] K. E. Freese, L. Kokai, R. P. Edwards et al., "Adipose-derived stems cells and their role in human cancer development, growth, progression, and metastasis: a systematic review," Cancer Research, vol. 75, no. 7, pp. 1161-1168, 2015.

[2] A. L. Strong, M. E. Burow, J. M. Gimble, and B. A. Bunnell, "Concise review: the obesity cancer paradigm: exploration of the interactions and crosstalk with adipose stem cells," Stem Cells, vol. 33, no. 2, pp. 318-326, 2015.

[3] A. Orimo, P. B. Gupta, D. C. Sgroi et al., "Stromal fibroblasts present in invasive human breast carcinomas promote tumor growth and angiogenesis through elevated SDF-1/CXCL12 secretion," Cell, vol. 121, no. 3, pp. 335-348, 2005.

[4] S. Kidd, E. Spaeth, K. Watson et al., "Origins of the tumor microenvironment: quantitative assessment of adiposederived and bone marrow-derived stroma," PLoS One, vol. 7, no. 2, article e30563, 2012.

[5] M. Augsten, "Cancer-associated fibroblasts as another polarized cell type of the tumor microenvironment," Frontiers in Oncology, vol. 4, p. 62, 2014.

[6] S. Pinilla, E. Alt, F. J. Abdul Khalek et al., "Tissue resident stem cells produce CCL5 under the influence of cancer cells and thereby promote breast cancer cell invasion," Cancer Letters, vol. 284, no. 1, pp. 80-85, 2009.

[7] G. S. Karagiannis, T. Poutahidis, S. E. Erdman, R. Kirsch, R. H. Riddell, and E. P. Diamandis, "Cancer-associated fibroblasts drive the progression of metastasis through both paracrine and mechanical pressure on cancer tissue," Molecular Cancer Research, vol. 10, no. 11, pp. 1403-1418, 2012.

[8] N. Erez, S. Glanz, Y. Raz, C. Avivi, and I. Barshack, "Cancer associated fibroblasts express pro-inflammatory factors in human breast and ovarian tumors," Biochemical and Biophysical Research Communications, vol. 437, no. 3, pp. 397-402, 2013.

[9] D. Raman, P. J. Baugher, Y. M. Thu, and A. Richmond, "Role of chemokines in tumor growth," Cancer Letters, vol. 256, no. 2, pp. 137-165, 2007.

[10] P. Cirri and P. Chiarugi, "Cancer-associated-fibroblasts and tumour cells: a diabolic liaison driving cancer progression," Cancer Metastasis Reviews, vol. 31, no. 1-2, pp. 195-208, 2012.

[11] E. M. Chandler, B. R. Seo, J. P. Califano et al., "Implanted adipose progenitor cells as physicochemical regulators of breast cancer," Proceedings of the National Academy of Sciences of the United States of America, vol. 109, no. 25, pp. 9786-9791, 2012.

[12] C. Jotzu, E. Alt, G. Welte et al., "Adipose tissue derived stem cells differentiate into carcinoma-associated fibroblast-like cells under the influence of tumor derived factors," Cellular Oncology, vol. 34, no. 1, pp. 55-67, 2011.

[13] E. Devarajan, Y. H. Song, S. Krishnappa, and E. Alt, "Epithelialmesenchymal transition in breast cancer lines is mediated through PDGF-D released by tissue-resident stem cells," International Journal of Cancer, vol. 131, no. 5, pp. 1023-1031, 2012.

[14] V. Eterno, A. Zambelli, L. Pavesi et al., "Adipose-derived mesenchymal stem cells (ASCs) may favour breast cancer recurrence via $\mathrm{HGF} / \mathrm{c}-\mathrm{met}$ signaling," Oncotarget, vol. 5, no. 3, pp. 613-633, 2014.

[15] F. L. Muehlberg, Y. H. Song, A. Krohn et al., "Tissue-resident stem cells promote breast cancer growth and metastasis," Carcinogenesis, vol. 30, no. 4, pp. 589-597, 2009.

[16] B. G. Rowan, J. M. Gimble, M. Sheng et al., "Human adipose tissue-derived stromal/stem cells promote migration and early metastasis of triple negative breast cancer xenografts," PLoS One, vol. 9, no. 2, article e89595, 2014.

[17] G. Welte, E. Alt, E. Devarajan, S. Krishnappa, C. Jotzu, and Y. H. Song, "Interleukin-8 derived from local tissue-resident stromal cells promotes tumor cell invasion," Molecular Carcinogenesis, vol. 51, no. 11, pp. 861-868, 2012.

[18] L. Zimmerlin, A. D. Donnenberg, J. P. Rubin, P. Basse, R. J. Landreneau, and V. S. Donnenberg, "Regenerative therapy and cancer: in vitro and in vivo studies of the interaction between adipose-derived stem cells and breast cancer cells from clinical isolates," Tissue Engineering Part A, vol. 17, no. 1-2, pp. 93-106, 2011.

[19] M. Walter, S. Liang, S. Ghosh, P. J. Hornsby, and R. Li, "Interleukin 6 secreted from adipose stromal cells promotes migration and invasion of breast cancer cells," Oncogene, vol. 28, no. 30, pp. 2745-2755, 2009.

[20] A. R. Carmichael, "Obesity and prognosis of breast cancer," Obesity Reviews, vol. 7, no. 4, pp. 333-340, 2006.

[21] A. R. Carmichael, "Obesity as a risk factor for development and poor prognosis of breast cancer," BJOG, vol. 113, no. 10, pp. 1160-1166, 2006. 
[22] K. L. Spalding, E. Arner, P. O. Westermark et al., "Dynamics of fat cell turnover in humans," Nature, vol. 453, no. 7196, pp. 783-787, 2008.

[23] R. Monteiro and I. Azevedo, "Chronic inflammation in obesity and the metabolic syndrome," Mediators of Inflammation, vol. 2010, Article ID 289645, 10 pages, 2010.

[24] B. Oñate, G. Vilahur, S. Camino-López et al., "Stem cells isolated from adipose tissue of obese patients show changes in their transcriptomic profile that indicate loss in stemcellness and increased commitment to an adipocyte-like phenotype," BMC Genomics, vol. 14, no. 1, p. 625, 2013.

[25] A. L. Strong, A. C. Bowles, R. M. Wise et al., "Human adipose stromal/stem cells from obese donors show reduced efficacy in halting disease progression in the experimental autoimmune encephalomyelitis model of multiple sclerosis," Stem Cells, vol. 34, no. 3, pp. 614-626, 2016.

[26] A. L. Strong, T. A. Strong, L. V. Rhodes et al., "Obesity associated alterations in the biology of adipose stem cells mediate enhanced tumorigenesis by estrogen dependent pathways," Breast Cancer Research, vol. 15, no. 5, article R102, 2013.

[27] A. L. Strong, J. F. Ohlstein, B. A. Biagas et al., "Leptin produced by obese adipose stromal/stem cells enhances proliferation and metastasis of estrogen receptor positive breast cancers," Breast Cancer Research, vol. 17, no. 1, p. 112, 2015.

[28] G. Yu, X. Wu, M. A. Dietrich et al., "Yield and characterization of subcutaneous human adipose-derived stem cells by flow cytometric and adipogenic mRNA analyzes," Cryotherapy, vol. 12, no. 4, pp. 538-546, 2010.

[29] M. F. Gregor and G. S. Hotamisligil, "Inflammatory mechanisms in obesity," Annual Review of Immunology, vol. 29, no. 1, pp. 415-445, 2011.

[30] C. Jotzu, E. Alt, G. Welte et al., "Adipose tissue-derived stem cells differentiate into carcinoma-associated fibroblast-like cells under the influence of tumor-derived factors," Analytical Cellular Pathology, vol. 33, no. 2, pp. 61-79, 2010.

[31] K. Shiga, M. Hara, T. Nagasaki, T. Sato, H. Takahashi, and H. Takeyama, "Cancer-associated fibroblasts: their characteristics and their roles in tumor growth," Cancer, vol. 7, no. 4, pp. 2443-2458, 2015.

[32] A. L. Strong, J. A. Semon, T. A. Strong et al., "Obesity-associated dysregulation of calpastatin and MMP-15 in adiposederived stromal cells results in their enhanced invasion," Stem Cells, vol. 30, no. 12, pp. 2774-2783, 2012.

[33] C. Dethlefsen, G. Hojfeldt, and P. Hojman, "The role of intratumoral and systemic IL-6 in breast cancer," Breast Cancer Research and Treatment, vol. 138, no. 3, pp. 657664, 2013.

[34] Z. T. Schafer and J. S. Brugge, "IL-6 involvement in epithelial cancers," The Journal of Clinical Investigation, vol. 117, no. 12, pp. 3660-3663, 2007.

[35] A. Ray, K. J. Nkhata, and M. P. Cleary, "Effects of leptin on human breast cancer cell lines in relationship to estrogen receptor and HER2 status," International Journal of Oncology, vol. 30, no. 6, pp. 1499-1509, 2007.

[36] G. Soria and A. Ben-Baruch, "The inflammatory chemokines CCL2 and CCL5 in breast cancer," Cancer Letters, vol. 267, no. 2, pp. 271-285, 2008.

[37] Y. J. Li, G. Liu, Y. Li et al., “ $m d a-7 /$ IL-24 expression inhibits breast cancer through upregulation of growth arrest-specific gene 3 (gas 3 ) and disruption of $\beta 1$ integrin function," Molecular Cancer Research, vol. 11, no. 6, pp. 593-603, 2013.
[38] M. Zheng, D. Bocangel, B. Doneske et al., "Human interleukin 24 (MDA-7/IL-24) protein kills breast cancer cells via the IL-20 receptor and is antagonized by IL-10," Cancer Immunology, Immunotherapy, vol. 56, no. 2, pp. 205-215, 2007.

[39] P. Sansone, G. Storci, S. Tavolari et al., "IL-6 triggers malignant features in mammospheres from human ductal breast carcinoma and normal mammary gland," The Journal of Clinical Investigation, vol. 117, no. 12, pp. 3988-4002, 2007.

[40] D. Conze, L. Weiss, P. S. Regen et al., "Autocrine production of interleukin 6 causes multidrug resistance in breast cancer cells," Cancer Research, vol. 61, no. 24, pp. 8851-8858, 2001.

[41] S. Lin, Z. Gan, K. Han, Y. Yao, and D. Min, "Interleukin-6 as a prognostic marker for breast cancer: a meta-analysis," Tumori, vol. 101, no. 5, pp. 535-541, 2015.

[42] R. C. Baxter, "IGF binding proteins in cancer: mechanistic and clinical insights," Nature Reviews Cancer, vol. 14, no. 5, pp. 329-341, 2014. 

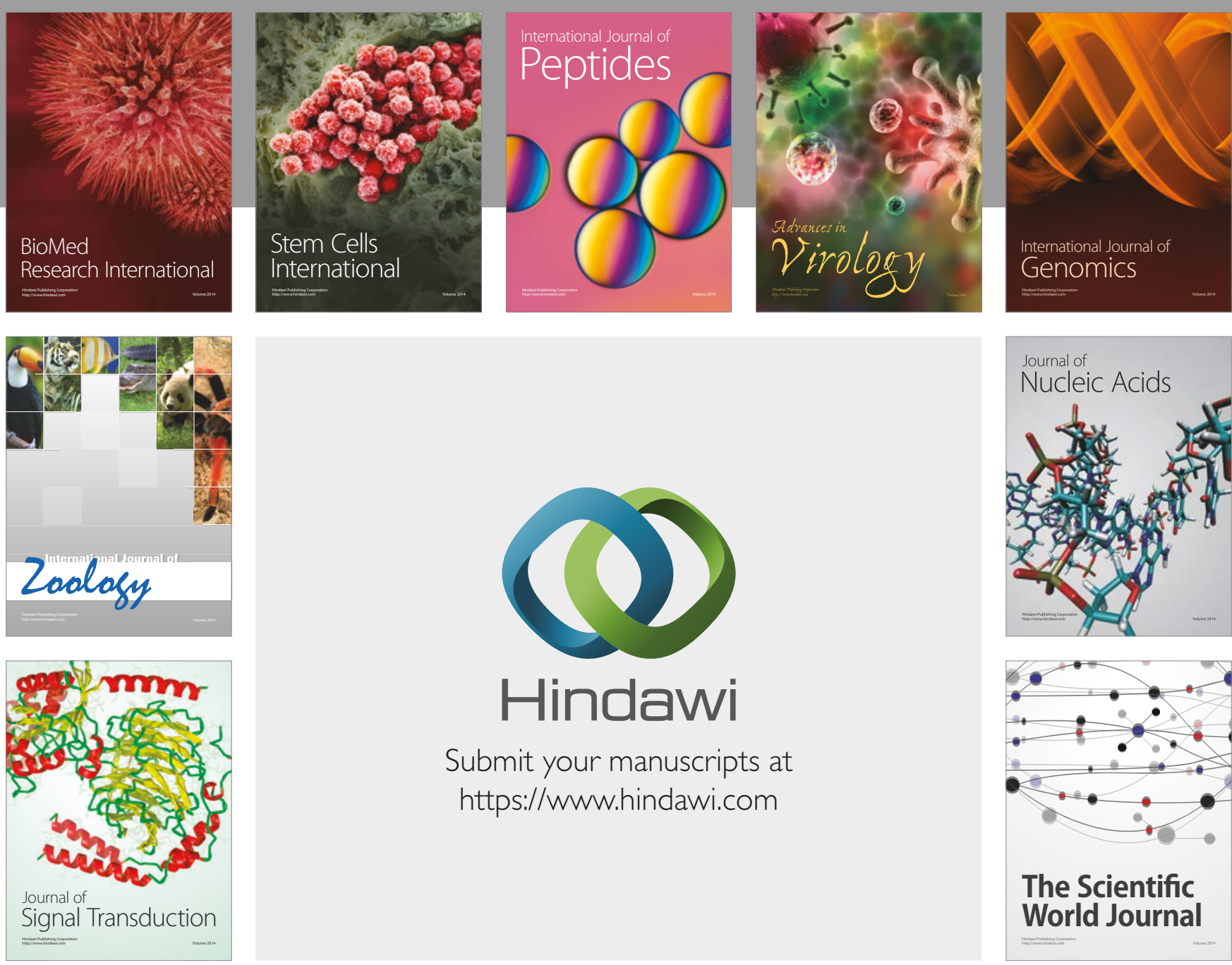

Submit your manuscripts at

https://www.hindawi.com
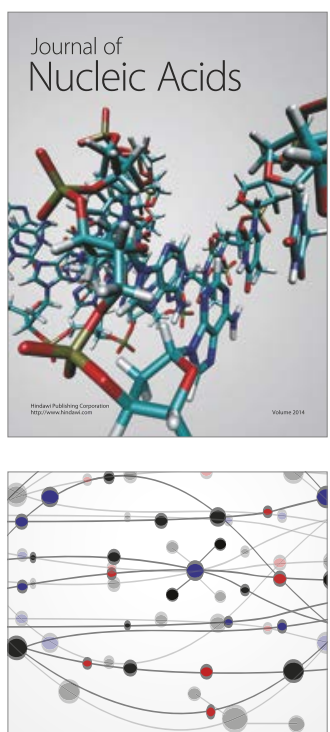

The Scientific World Journal

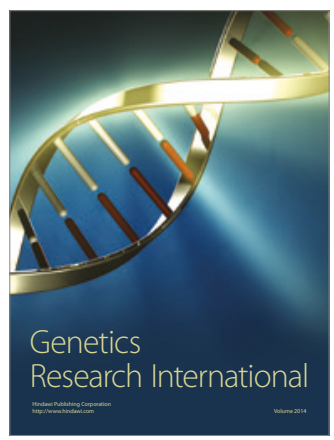

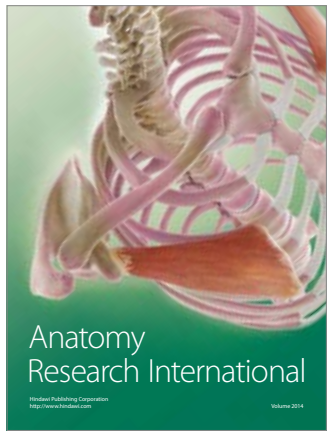

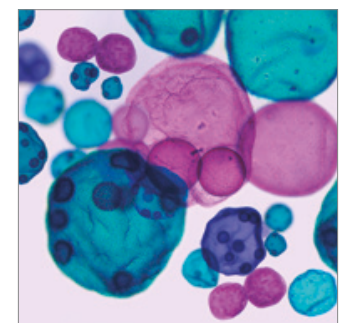

International Journal of Microbiology
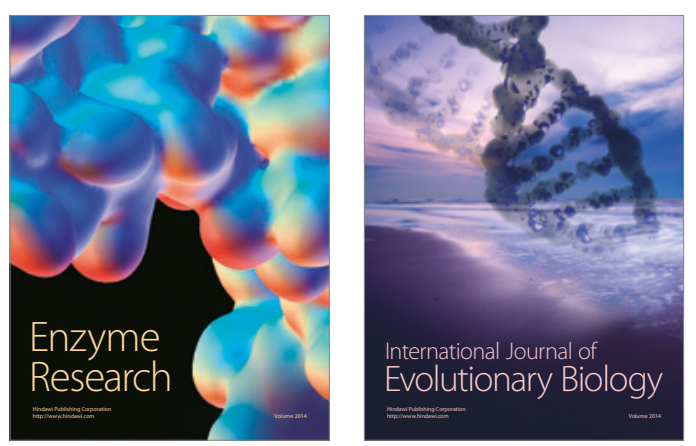
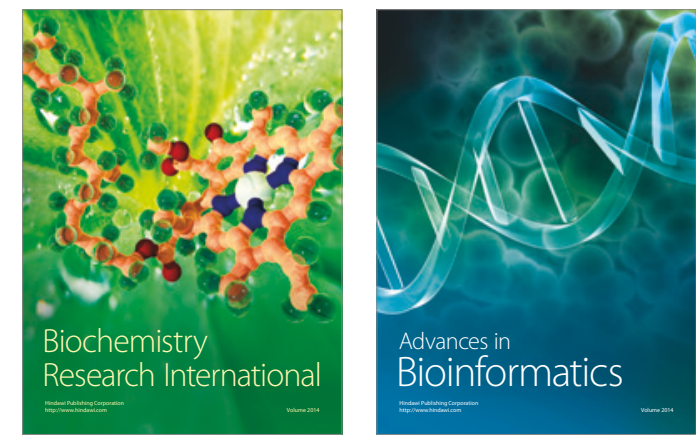

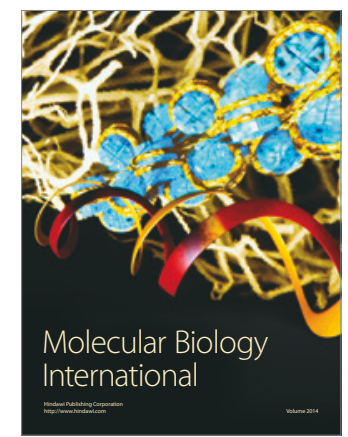

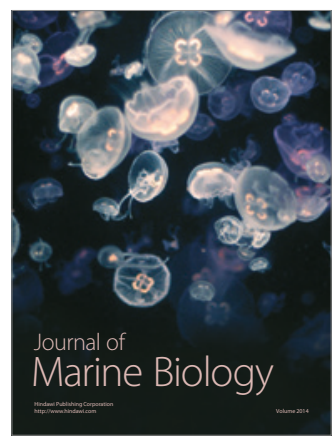

